\title{
Benchmarking real-valued acts
}

\author{
Erio Castagnoli ${ }^{\mathrm{a}}$, Marco LiCalzi ${ }^{\mathrm{b}, *}$ \\ ${ }^{a}$ Institute of Quantitative Methods, Bocconi University, Milan, Italy \\ ${ }^{\mathrm{b}}$ Department of Applied Mathematics, University of Venice, Dorsoduro 3825/e, 30123 Venezia, Italy \\ Received 28 January 2005 \\ Available online 16 November 2005
}

\begin{abstract}
A benchmarking procedure ranks real-valued acts by the probability that they outperform a benchmark $\beta$ which may itself be a random variable; that is, an act $f$ is evaluated by means of the functional $V(f)=$ $P(f \geqslant \beta)$. Expected utility is a special case of benchmarking procedure, where the acts and the benchmark are stochastically independent. This paper provides axiomatic characterizations of preference relations that are representable as benchmarking procedures. The key axiom is the sure-thing principle. When the state space is infinite, different continuity assumptions translate into different properties of the probability $P$. (C) 2005 Elsevier Inc. All rights reserved.
\end{abstract}

JEL classification: D81

Keywords: Sure-thing principle; State-dependent expected utility; Measure representation approach; Target-based reasoning

\section{Introduction}

A classical problem in decision theory under uncertainty is to rank a set of acts defined on a state space $S$. The standard approach is to axiomatize a preference relation representable by means of a functional, which is in turn used as a convenient tool to construct a consistent ranking. These axiomatizations take the state space $S$ as a primitive. However, there exist natural situations where the agent may wish to consider a larger state space $\Omega$ in order to rank the acts over $S$. Consider the following example.

\footnotetext{
* Corresponding author. Fax: (+39) 0415221756.

E-mail address: licalzi@unive.it (M. LiCalzi).
} 
Julia is looking at several alternative long-term investments for her retirement. She wishes to maximize the probability to raise enough money and meet her future needs. Let $f$ and $\beta$ represent the money raised by an investment and the amount needed by Julia, respectively. At this time, both are uncertain quantities that we can view as acts. The payoff $f$ depends on events such as the business cycle, or the rate of technological innovation; hence, the natural state space $S$ for the investments should list these economic variables. The $\operatorname{sum} \beta$ that Julia is going to need at retirement depends also on noneconomic variables such as her health conditions or the age of her descendants, which may not be listed in $S$. Therefore, if Julia wishes to rank an investment by the probability $P(f \geqslant \beta)$ that it meets her retirements needs, she needs to expand the set $S$ to a larger set $\Omega$.

This example illustrates the following general ranking procedure. Given a set $\mathcal{F}$ of acts on a state space $S$, construct a benchmark $\beta$ defined on a larger set $\Omega$ and a probability measure $\mathbb{P}$ on $\Omega$. Then, rank an act $f$ in $\mathcal{F}$ by the probability $\mathbb{P}(f \geqslant \beta)$ that it outperforms the benchmark. We call this procedure benchmarking, in analogy with the practice to measure a rival's product by a set of specified standards and compare it with one's own product. Throughout this paper, benchmarking denotes an evaluation procedure that ranks a set of options by the probability that they meet a given target. See Bordley (2002). The benchmarking procedure is more general than it may appear: we show in Section 3.2 that it includes expected utility as a special case.

Of course, one can apply the existing axiomatizations for preference representation to the benchmarking procedure simply by expanding the primitive state space to $\Omega$. This is a natural move and, in a well-known discussion about small worlds, Savage (1954) recommends that the state space be taken as large as needed to include all relevant events. However, this approach has two serious shortcomings. It increases the complexity of the decision problem, because it expands the set of acts that needs to be ranked. And it forces the agent to express preferences over imaginary acts, such as-in Julia's example-long-term investments whose payoff depends on the recipient's health conditions. Sometimes, it may be desirable to keep the domain of the acts as small as possible.

The purpose of this paper is to characterize the benchmarking procedure when acts are defined only on the smaller state space $S$. Technically, the problem is to simultaneously construct a benchmark $\beta$ and a probability measure $\mathbb{P}$ such that a preference relation $\succeq$ on $\mathcal{F}$ is represented by the functional $V(f)=\mathbb{P}(f \geqslant \beta)$. We solve this problem under the structural assumption that the benchmark is defined on a distinct state space $S^{\prime}$ and that $\Omega=S \times S^{\prime}$.

Assuming that the space $\Omega$ is a Cartesian product requires that the events affecting the benchmark $\beta$ are different from those affecting the available acts in $\mathcal{F}$; i.e., the acts and the benchmark should be logically separate. In Julia's example, for instance, this would imply that the economic variables associated with $S$ are not relevant to determine the amount $\beta$ she needs at retirement. This logical separability between the state spaces $S$ and $S^{\prime}$ implies no a priori restrictions for the probability measure $\mathbb{P}$ on their Cartesian product $\Omega=S \times S^{\prime}$. In particular, the benchmark $\beta$ and the acts in $\mathcal{F}$ may exhibit stochastic dependence in general.

Under the structural assumption $\Omega=S \times S^{\prime}$, the benchmarking procedure turns out to be closely related to the additively decomposable representation known as state-dependent expected utility. When the state space $S$ is finite, axiomatizations of this representation are well-known; see Debreu (1960) and Gorman (1968). Recently, Wakker and Zank (1999) have shown how to extend the representation to the case where $S$ is not finite. Assuming the supnorm continuity of preferences, they prove the existence of a representing functional for all bounded measurable acts, but derive an explicit representation only over simple acts. Instead, the benchmarking approach generates an explicit representation for all bounded measurable acts. In this respect, 
another contribution of the paper is to offer a single comprehensive representation for different classes of additively decomposable preferences.

In particular, we characterize standard (i.e., state-independent) expected utility as the benchmarking procedure associated with the benchmark being stochastically independent of the acts. The formal equivalence of these two models was discussed in Castagnoli and LiCalzi (1996) with reference to preferences over lotteries in a von Neumann and Morgenstern setting. They used this observation to reinterpret several known results and suggest a few new ones. Later, Bordley and LiCalzi (2000) carried out a similar exercise with reference to preferences over acts in a Savage setting.

From a technical point of view, this paper relies heavily on an approach that derives representation theorems by measuring sets associated with functions. This idea appeared first in Segal (1989). He suggested to evaluate the distribution functions of real-valued random variables by a measure defined over their epigraphs and dubbed this as the "measure representation" approach. A few papers followed in this vein until Wakker (1993) pointed out an error in the statement of the representation theorem, which was amended in Segal (1993). Subsequently, a few papers have extended the measure representation approach to different environments. LiCalzi (1998) applies it to real-valued functions of bounded variation and Chateauneuf (1999) to probability distributions over a connected compact space. The papers closest to ours are Chew and Wakker (1996) and especially Wakker and Zank (1999), which generalize the axiomatics underlying the measure representation approach from probability distributions to acts. These two papers cast their results in a functional language; therefore, properly speaking, they do not interpret their representing functionals as measures on some collection of sets.

The paper is organized as follows. Section 2 collects notation and preliminaries. Section 3 characterizes the benchmarking procedure for a finite state space. In particular, Section 3.2 characterizes the special case in which the benchmarking procedure is equivalent to the standard expected utility model. Section 4 extends the characterizations to the case where the state space is not finite. Herein, three different results record the subtle influence of increasingly stronger assumptions on the continuity of the preference relation. Appendix A gives additional background on the extensions of measures. Appendix B collects all proofs.

\section{Setup and notation}

\subsection{Measure-theoretic preliminaries}

We are interested in families of sets with different closure properties. An algebra $\mathcal{A}$ on a set $S$ is a nonempty collection of subsets of $S$ that is closed under complementation and finite unions; clearly, an algebra on $S$ includes $S$ itself. A $\sigma$-algebra $\sigma \mathcal{A}$ on $S$ is an algebra that is closed under countable unions.

The notion of algebra admits two distinct generalizations. A semialgebra $\mathcal{S}$ on $S$ is a collection of subsets of $S$ that includes $S$ and the empty set $\emptyset$, is closed under finite intersections, and for any $A, B \in \mathcal{S}$ contains pairwise disjoint sets $C_{1}, \ldots, C_{n}$ such that $A \backslash B=\bigcup_{i=1}^{n} C_{i}$. A lattice $\mathcal{L}$ on $S$ is a collection of subsets of $S$ that includes $S$ and is closed under finite unions and finite intersections.

If $\mathcal{S}_{1}$ and $\mathcal{S}_{2}$ are two semialgebras on $S_{1}$ and $S_{2}$ respectively, the family of rectangles $\{A \times B$ : $\left.A \in \mathcal{S}_{1}, B \in \mathcal{S}_{2}\right\}$ is a semialgebra of subsets on $S_{1} \times S_{2}$ called the product semialgebra. The product semialgebra is denoted by $\mathcal{S}_{1} \times \mathcal{S}_{2}$. Even if $\mathcal{S}_{1}$ and $\mathcal{S}_{2}$ are $\sigma$-algebras, the product semi- 
algebra $\mathcal{S}_{1} \times \mathcal{S}_{2}$ need not be an algebra. The smallest algebra and $\sigma$-algebra generated by the product semialgebra $\mathcal{S}_{1} \times \mathcal{S}_{2}$ are denoted by $\mathcal{A}\left(\mathcal{S}_{1} \times \mathcal{S}_{2}\right)$ and $\sigma \mathcal{A}\left(\mathcal{S}_{1} \times \mathcal{S}_{2}\right)$, respectively.

Given an arbitrary collection $\mathcal{C}$ of subsets of $S$, consider a real-valued set function $m$ on $\mathcal{C}$. We say that $m$ is grounded if $\emptyset \in \mathcal{C}$ and $m(\emptyset)=0$. It is positive if $m(A) \geqslant 0$ for all $A$ in $\mathcal{C}$. It is additive if for each pair $A, B$ of disjoint sets in $\mathcal{C}$ with $A \cup B$ in $\mathcal{C}$, we have $m(A \cup B)=$ $m(A)+m(B)$. It is $\sigma$-additive or countably additive if the equality $m\left(\bigcup_{i=1}^{\infty} A_{i}\right)=\sum_{i=1}^{\infty} m\left(A_{i}\right)$ holds for any sequence $\left\{A_{i}\right\}$ of pairwise disjoint sets. We say that $m$ is finitely additive when it is additive but not necessarily countably additive. Finally, a grounded set function $m$ is strongly additive if for each pair $A, B$ in $\mathcal{C}$ with $A \cap B$ and $A \cup B$ in $\mathcal{C}$, we have $m(A)+m(B)=$ $m(A \cap B)+m(A \cup B)$.

A real-valued set function $m$ over a semialgebra $\mathcal{S}$ on $S$ is a finitely (or countably) additive measure if it is grounded, positive, and finitely (or countably) additive. The definition obviously extends to algebras or $\sigma$-algebras. We call $(\sigma$-)measurable space any pair $(S, \Sigma)$ where $S$ is a set and $\Sigma$ is a $(\sigma-)$ algebra on $S$. A finitely (or countably) additive measure over a measurable space $(S, \Sigma)$ such that $m(S)=1$ is called a finitely (or countably) additive probability measure on $\mathcal{S}$. Finitely additive probability measures are also known as charges. A finitely additive measure $m$ on a measurable space $(S, \Sigma)$ is nonatomic if, for each subset $A$ in $\Sigma$ and each strictly positive $x<m(A)$, there exists another subset $B$ in $\Sigma$ such that $B \subseteq A$ and $m(B)=x$; a subset $A$ in $\Sigma$ is an atom if, for any $B \subseteq A, m(B) \cdot m(A \backslash B)=0$. Under finite additivity, nonatomicity implies the nonexistence of atoms; under countable additivity, the two are equivalent.

\subsection{Decision-theoretic preliminaries}

We consider a variant of the framework in Savage (1954). There is a state space $S^{o}$, which can be finite or infinite. (The superscript is a mnemonic for "overt" because this state space is a primitive of the model, and thus its description must be explicit.) It is endowed with an algebra $\Sigma^{o}$ of subsets which we call events. Therefore, $\left(S^{o}, \Sigma^{o}\right)$ is a measurable space. The outcome space $X$ is a compact interval $[a, b]$ of $\mathbb{R}$ with nonempty interior. ${ }^{1}$ An act is a measurable function $f: S^{o} \rightarrow X$ and $\mathcal{F}$ denotes the set of all acts. All elements of $\mathcal{F}$ inherit from $X$ the property of being bounded. An act is simple if it takes only finitely many values and $\mathcal{F}_{S}$ denotes the set of simple acts. Under the usual abuse of notation, we identify $X$ with the subset of constant acts in $\mathcal{F}$.

A preference relation is a binary relation $\succeq$ on $\mathcal{F}$. As customary, we denote by $\sim$ and $\succ$ its symmetric and asymmetric components. A functional $V: \mathcal{F} \rightarrow \mathbb{R}$ represents $\succeq$ whenever $V(f) \geqslant V(g)$ if and only if $f \succeq g$. A necessary condition for $\succeq$ to have a representation is that it is a weak order; i.e., a complete and transitive relation.

Given two acts $f$ and $g$ and an event $A$, let $f A g$ denote the act which agrees with $f$ on $A$ and with $g$ on $A^{c}$. The restriction of an act $f$ to an event $A$ is denoted by $f_{A}$. An event $A$ is null if $f A g \sim g$ for all simple acts $f$ and $g$; an event which is not null is essential. A preference relation $\succeq$ is strictly monotone if $x A f \succ y A f$ for all nonnull events $A$, simple acts $f$ and outcomes $x>y$. The preference relation $\succeq$ satisfies Savage's sure-thing principle whenever $f A h \succeq g A h$ if and only if $f A h^{\prime} \succeq g A h^{\prime}$ for all acts $f, g, h, h^{\prime}$ and events $A$. When this condition is imposed only on simple acts, we say that the sure-thing principle holds on $\mathcal{F}_{s}$.

\footnotetext{
1 This compactness assumption is not innocuous. It ensures that the (continuous) utility functions in our representations have a compact range. Dispensing with it leads to two possible developments. One introduces additional axioms that imply compact-ranged utilities. The other one relaxes the requirement that the benchmarking measure be finite.
} 


\section{Benchmarking over a finite state space}

We aim to provide a behavioral characterization of the benchmarking procedure for acts defined on a measurable space $\left(S^{o}, \Sigma^{o}\right)$. That is, we want to derive a set of axioms for a preference relation $\succeq$ on a set of acts such that $f \succeq g$ if and only if $\mathbb{P}(f \geqslant \beta) \geqslant \mathbb{P}(g \geqslant \beta)$, where $\mathbb{P}$ is a probability measure and $\beta$ is a benchmark. We assume that the benchmark $\beta$ is defined on a distinct measurable space $\left(S^{c}, \Sigma^{c}\right)$. The superscript is a mnemonic for "covert" because this state space is not a primitive of the model: its existence is endogenously derived as part of the representation.

Formally, we derive simultaneously the existence of a benchmark $\beta$ on $S^{c}$ and of a unique probability measure $\mathbb{P}$ on the space $\Omega=S^{o} \times S^{c}$ such that a given preference relation $\succeq$ on $\mathcal{F}$ is represented by the functional $V(f)=\mathbb{P}(f \geqslant \beta)$. We denote by $\mathbb{P}^{o}$ and $\mathbb{P}^{c}$ the marginal probability measures on $S^{o}$ and $S^{c}$ induced by $\mathbb{P}$; for brevity, we call these two probability measures the "marginals."

This section studies the case where the (overt) state space $S^{o}$ is finite. This is the natural setting for most applications in decision analysis, where the benchmarking procedure provides a useful language for structuring an agent's ranking. The representation results for a finite state space obtain also as corollaries of our results for a general state space, but a separate presentation helps to make explicit the different strategy of proof and the technical hurdles associated with a general state space.

\subsection{General benchmarking}

Throughout this section, we assume that $S^{o}=\left\{s_{1}, \ldots, s_{i}, \ldots, s_{n}\right\}$ is a finite set. Then all acts are simple and $\mathcal{F}=\mathcal{F}_{s}$; moreover, we can identify $\mathcal{F}$ with the subset $[a, b]^{n}$ of $\mathbb{R}^{n}$. We say that the preference relation $\succeq$ is continuous if, for each act $f$, the upper level set $\{g: g \succeq f\}$ and the lower level set $\{g: f \succeq g\}$ are closed subsets of $\mathbb{R}^{n}$ in the Euclidean topology. The following result states that, under mild technical assumptions, a benchmarking representation is possible if and only if preferences satisfy the sure-thing principle. The main technical assumption is that $S^{o}$ has at least three essential states; note that the case where $S^{o}$ has exactly one essential state is trivial, because there is no uncertainty. The case with only two states requires an additional "hexagon condition" over preferences; see Karni and Safra (1998) and the comment in Köbberling (2003).

Theorem 1. Suppose that $S^{o}=\left\{s_{1}, \ldots, s_{i}, \ldots, s_{n}\right\}$ has at least three essential states. Then the following three statements are equivalent.

(i) The weak order $\succeq$ is strictly monotone, continuous, and satisfies the sure-thing principle.

(ii) There exist continuous functions $U_{i}: X \rightarrow[0,1]$ for $i=1, \ldots, n$ that are strictly increasing for all essential states and constant for all null states, such that $\succeq$ is represented by

$$
V(f)=\sum_{i=1}^{n} U_{i}\left(f\left(s_{i}\right)\right) .
$$

(iii) There exist a (covert) measurable space $\left(S^{c}, \Sigma^{c}\right)$, a finitely additive probability measure $\mathbb{P}$ over the algebra $\mathcal{A}\left(\Sigma^{o} \times \Sigma^{c}\right)$ on $S^{o} \times S^{c}$, and an injective benchmark $\beta: S^{c} \rightarrow[a, b]$, such that $\succeq$ is represented by

$$
V(f)=\mathbb{P}(f \geqslant \beta) \text {. }
$$


Moreover, the probability measure $\mathbb{P}$ is unique; the marginal $\mathbb{P}^{o}$ on $S^{o}$ has $\mathbb{P}^{o}\left(s_{i}\right)>0$ if and only if a state $s_{i}$ is essential; and the marginal $\mathbb{P}^{c}$ on $S^{c}$ (and hence $\mathbb{P}$ itself) are nonatomic.

Parts (i) and (ii) state the well-known result in Debreu (1960) that the sure-thing principle is equivalent to the existence of an additively decomposable representation, also known as the statedependent expected utility model. Part (iii) states the formal equivalence between this model and the benchmarking procedure over a state space $\Omega=S^{o} \times S^{c}$. The proof of (iii) runs in two steps. First, we "hijack" Debreu's theorem to derive a benchmarking measure $\mathbb{P}$ over the rectangles in $S^{o} \times S^{c}$. Second, we show that the existence of a benchmarking representation is equivalent to additive decomposability. By relying on Debreu's result, the proof of Theorem 1 adheres as much as possible to standard techniques in decision theory.

Given the covert measurable space $\left(S^{c}, \Sigma^{c}\right)$, the probability measure $\mathbb{P}$ is unique in the sense that a benchmarking measure $m$ such that $V(f)=m(f \geqslant \beta)$ represents $\succeq$ must be a positive rescaling of $\mathbb{P}$. In other words, $\mathbb{P}$ is the only benchmarking measure which is also a probability measure. This uniqueness mirrors the well-known result that the representation in Theorem 1(ii) is unique up to cardinal transformations. More precisely, another functional $W(f)=\sum_{i} W_{i}\left(f\left(s_{i}\right)\right)$ represents $\succeq$ if and only if there exist real numbers $\alpha_{1}, \ldots, \alpha_{n}$ and a strictly positive $\gamma$ such that $W_{i}=\alpha_{i}+\gamma U_{i}$ for all $i$, implying that $W=\sum_{i} \alpha_{i}+\gamma V$. In the course of the proof, we use up all degrees of freedom by normalizing $V$ so that $V(a)=0$ and $V(b)=1$.

The injectivity of the benchmark implies that the covert state space $S^{c}$ has minimal cardinality. Given that the strict monotonicity of $\succeq$ implies that the range of $\beta$ must be $[a, b]$, we actually set $S^{c}=[a, b]$. This choice seems the natural one. Since we are only given information about preferences over acts defined on $S^{o}$, we cannot learn anything substantial on the covert state space $S^{c}$. Yet, benchmarking requires a summary description of the payoff-relevant events which were outside the original scope of the decision problem underlying $S^{o}$. Choosing $S^{c}=[a, b]$ provides this summary, because it amounts to mapping all the external events leading to a payoff $x$ to the point $x$ itself. Thus, although the agent might have a different covert state space in mind, $S^{c}=[a, b]$ is a parsimonious choice which captures all relevant interactions.

Finally, it is worthwhile to note that the benchmarking measure $\mathbb{P}$ induces a marginal probability measure $\mathbb{P}^{o}$ on the measurable space $\left(S^{o}, \Sigma^{o}\right)$ which attaches strictly positive weights only to essential events. Therefore, the benchmarking measure is consistent with the standard interpretation that the agent has a subjective probability describing his beliefs over the likelihoods of the (overt) events.

\subsection{Independent benchmarking}

For the measure representation approach over probability distributions, Segal (1989) shows that we end up with the expected utility representation when the measure over epigraphs can be written as the product of the two marginal measures. This strongly suggests that, under the benchmarking approach, we should recover the standard (state-independent) expected utility representation over acts when the probability measure $\mathbb{P}$ is the product of the two marginal probability measures $\mathbb{P}^{O}$ and $\mathbb{P}^{c}$. Inspired by this, we call independent benchmarking the case where stochastic independence between the acts and the benchmark holds. As we show momentarily, under this assumption the benchmarking procedure is formally equivalent to ranking acts by their expected utility. See Bordley and LiCalzi (2000). 
We need an additional definition. A preference relation $\succeq$ satisfies tradeoff consistency if $x A f \sim y A g, w A f \sim z A g$, and $x B h \sim y B h^{\prime}$ imply $w B h \sim z B h^{\prime}$ for all outcomes $x, y, w, z$, simple acts $f, g, h, h^{\prime}$ and essential events $A, B$. Tradeoff consistency requires that, when preferences reveal that $x$ compares to $y$ as favorably as $w$ compares to $z$ on the essential event $A$, this pattern stays unchanged over another essential event $B$.

The first (and stronger) version of tradeoff consistency was introduced in Wakker (1984). Köbberling and Wakker (2003) thoroughly discusses alternative weaker formulations, including the present one. For our purposes, the main advantage of tradeoff consistency is that it offers a clean way to a characterization as close to Theorem 1 as possible. The next result states that, under mild technical assumptions, an independent benchmarking representation is possible if and only if preferences satisfy tradeoff consistency.

Theorem 2. Suppose that $S^{o}$ has at least three essential states. Then the following three statements are equivalent,

(i) The weak order $\succeq$ is strictly monotone, continuous, and satisfies tradeoff consistency.

(ii) There exist a continuous strictly increasing function $U: X \rightarrow[0,1]$ and a finitely additive probability measure $P$ on $\left(S^{o}, \Sigma^{o}\right)$, such that $\succeq$ is represented by

$$
V(f)=\sum_{i=1}^{n} U\left(f\left(s_{i}\right)\right) \cdot P\left(s_{i}\right) .
$$

Moreover, $P\left(s_{i}\right)>0$ if and only if a state $s_{i}$ is essential.

(iii) The statement in Theorem 1(iii) holds. Moreover, $\mathbb{P}=\mathbb{P}^{o} \times \mathbb{P}^{c}$ can be factored in the product of the two marginals; i.e., stochastic independence holds.

The equivalence of (i) and (ii) is a minor variant of Corollary 10 in Köbberling and Wakker (2003). The equivalence of (ii) and (iii) states the formal equivalence of independent benchmarking and standard expected utility. Roughly speaking, the state independence of the von Neumann and Morgenstern utility function $U(x)$ in the expected utility model is equivalent to the stochastic independence of the benchmark $\beta$ from the acts in $\mathcal{F}$ in the benchmarking procedure. An analogous result for preferences over probability distributions appears in Castagnoli and LiCalzi (1996).

\section{Benchmarking over an infinite state space}

Throughout this section, we assume that the overt space $S^{o}$ is infinite. We provide three results, that rely on increasingly restrictive notions of continuity. Theorem 3 derives the benchmarking representation of Theorem 1 for simple acts over an infinite state space. Theorem 4 extends this representation to the set of all acts. Theorem 5 imposes the additional restriction that the benchmarking measure be countably additive. These three results hold in general for any space $S^{o}$ but, when $S^{o}$ is finite, they collapse to the same statement. Thus, restricting attention to an infinite state space $S^{o}$ is necessary to avoid trivialities.

\subsection{Simple continuity}

We need an additional definition. A preference relation $\succeq$ is simply continuous if, for each finite partition $\Pi=\left\{A_{1}, \ldots, A_{n}\right\} \subseteq \Sigma^{o}$ of the state space $S^{o}$, it is continuous over the finite- 
dimensional subspace of simple acts that are $\Pi$-measurable. A $\Pi$-measurable act $f$ is of the form $f=\sum_{i=1}^{n} x_{i} \mathbf{1}\left(A_{i}\right)$. Therefore, simple continuity requires that for every $\left(x_{1}, \ldots, x_{n}\right)$ in $\mathbb{R}^{n}$, the upper level set $\left\{\left(y_{1}, \ldots, y_{n}\right): \sum_{i=1}^{n} y_{i} \mathbf{1}\left(A_{i}\right) \succeq \sum_{i=1}^{n} x_{i} \mathbf{1}\left(A_{i}\right)\right\}$ and the lower level set $\left\{\left(y_{1}, \ldots, y_{n}\right): \sum_{i=1}^{n} x_{i} \mathbf{1}\left(A_{i}\right) \succeq \sum_{i=1}^{n} y_{i} \mathbf{1}\left(A_{i}\right)\right\}$ are closed subsets of $\mathbb{R}^{n}$ in the Euclidean topology. Simple continuity is equivalent to standard continuity of $\succeq$ when $S^{o}$ is finite, and in general it is substantially weaker than assuming continuity of $\succeq$ in the product topology.

The first result states that, after exchanging simple continuity for standard continuity, the benchmarking representation of Theorem 1 for arbitrary acts over a finite state space holds for simple acts over an infinite state space as well. Given a measurable space $\left(S^{o}, \Sigma^{o}\right)$, recall that an event $A$ is an element of the algebra $\Sigma^{o}$. Moreover, given a probability measure $\mathbb{P}$ over an algebra on $S^{o} \times S^{c}$, denote by $\mathbb{P}^{o}$ and $\mathbb{P}^{c}$ the marginal probability measures induced on $S^{o}$ and $S^{c}$, respectively.

Theorem 3. Suppose that $S^{o}$ has at least three essential disjoint events. Then the following three statements are equivalent.

(i) The weak order $\succeq$ is strictly monotone, simply continuous on $\mathcal{F}_{s}$, and satisfies the sure-thing principle on $\mathcal{F}_{s}$.

(ii) There exist continuous functions $U_{A}: X \rightarrow[0,1]$ for each event $A$ that are strictly increasing when $A$ is essential and constant when $A$ is null, such that $\succeq$ on $\mathcal{F}_{S}$ is represented by

$$
V\left(\sum_{i=1}^{n} x_{i} \mathbf{1}\left(A_{i}\right)\right)=\sum_{i=1}^{n} U_{A_{i}}\left(x_{i}\right) .
$$

(iii) There exist a (covert) measurable space $\left(S^{c}, \Sigma^{c}\right)$, a finitely additive probability measure $\mathbb{P}$ over the algebra $\mathcal{A}\left(\Sigma^{o} \times \Sigma^{c}\right)$ on $S^{o} \times S^{c}$, and an injective benchmark $\beta: S^{c} \rightarrow[a, b]$, such that $\succeq$ on $\mathcal{F}_{s}$ is represented by

$$
V(f)=\mathbb{P}(f \geqslant \beta) .
$$

Moreover, $\mathbb{P}$ is unique; $\mathbb{P}^{o}(A)>0$ if and only if the event $A$ is essential; and $\mathbb{P}^{c}$ on $S^{c}$ (and hence $\mathbb{P}$ itself) are nonatomic.

The equivalence of (i) and (ii) is a corollary of the additively decomposable representation in Debreu (1960) for finite state spaces, and appears as Proposition 3 in Wakker and Zank (1999). We use (ii) as a useful shortcut to derive the benchmarking measure $\mathbb{P}$ in (iii). The full proof of the equivalence between (ii) and (iii) is in Appendix B, but we offer hereafter a compact description. The measure-theoretic approach in the proof applies unchanged to the derivation of the next two results.

An act $f$ is a function from $S^{o}$ in $X=[a, b]$. Identify each simple act $f$ with its subgraph $\hat{f}=$ $\left\{(s, x) \in S^{o} \times[a, b]: a \leqslant x \leqslant f(s)\right\}$. There is an obvious $1-1$ onto mapping between the set $\mathcal{F}_{S}$ of simple acts and the set $\mathcal{G}_{0}$ of their subgraphs. Since $\mathcal{G}_{0}$ is closed with respect to finite unions and finite intersections, it is a lattice. A functional $V$ on $\mathcal{F}_{s}$ defines a set function $m$ on the lattice $\mathcal{G}_{0}$. In particular, it is possible to choose a version of the functional $V$ in Theorem 3(ii) that generates a set function $m$ which is grounded, positive, and strongly additive on the lattice $\mathcal{G}=\mathcal{G}_{0} \cup\{\emptyset\}$. This set function $m$ uniquely extends to a measure $m^{\prime}$ on the semialgebra $\mathcal{S}(\mathcal{G})$ generated by $\mathcal{G}$. This semialgebra coincides with the product semialgebra $\Sigma^{o} \times \Sigma^{c}$ and thus $m^{\prime}$ can be uniquely 
extended to a (finitely additive) probability measure $\mu$ on the algebra $\mathcal{A}\left(\Sigma^{o} \times \Sigma^{c}\right)$ on $S^{o} \times S^{c}$ generated by $\Sigma^{o} \times \Sigma^{c}$.

This method of proof works for any overt state space $S^{o}$ given as a primitive of the model. On the other hand, we enjoy some freedom in the choice of the covert measurable space $\left(S^{c}, \Sigma^{c}\right)$ that is derived as part of the representation. For instance, the measurable space $\left(S^{c}, \Sigma^{c}\right)$ may be replaced with another measurable space $\left(S_{1}^{c}, \Sigma_{1}^{c}\right)$ if there exists a 1-1 and $\left(\Sigma_{1}^{c}, \Sigma^{c}\right)$-measurable transformation between $\Sigma_{1}^{c}$ and $\Sigma^{c}$.

Our derivation of the benchmarking measure makes the minimal assumption that the "covert" state space $S^{c}$ is the interval $[a, b]$ itself. For consistency, in this section we keep the size of the corresponding algebra $\Sigma^{c}$ as small as possible and, differently from the proof of Theorem 1, we do not assume upfront that $\Sigma^{c}$ is the Borel $\sigma$-algebra $\mathcal{B}([a, b])$. This is without loss of generality, because it is always possible to complete $\Sigma^{c}$ into the $\sigma$-algebra $\mathcal{B}([a, b])$ and extend the (nonatomic) marginal probability measure $\mathbb{P}^{c}$ on $\Sigma^{c}$ to a countably additive measure on $\mathcal{B}([a, b])$. But we feel that these manipulations of the "covert" measurable space are not fundamental for the argument.

\subsection{Supnorm continuity}

In this section, we extend the benchmarking representation of Theorem 3 from simple acts to all acts. We need a few definitions. The preference relation $\succeq$ on the set $\mathcal{F}$ of all acts is supnorm continuous if, for each act $f$, the upper level set $\{g: g \succeq f, g \in \mathcal{F}\}$ and the lower level set $\{g: f \succeq g, g \in \mathcal{F}\}$ are closed subsets in the topology induced by the supnorm $\|f\|=\sup _{s}|f(s)|$. Supnorm continuity is equivalent to standard continuity of $\succeq$ when $S^{o}$ is finite, and it implies simple continuity.

A functional $V: \mathcal{F} \rightarrow \mathbb{R}$ is additively decomposable over disjoint events if for each event $A$ there exists a functional $U_{A}$ on the set $\mathcal{F}_{A}$ of restrictions $f_{A}$ of an act $f$ to $A$ such that, for each finite partition $\left\{A_{1}, \ldots, A_{n}\right\}$ of $S^{o}, V(f)=\sum_{i=1}^{n} U_{A_{i}}\left(f_{A_{i}}\right)$. The second result states that, after exchanging supnorm continuity for simple continuity, the benchmarking representation of Theorem 3(iii) extends from the set $\mathcal{F}_{s}$ of the simple acts to the set $\mathcal{F}$ of all acts. For symmetry and ease of comparison, we state in (ii) the analog of Theorem 3(ii) asserting the existence of a representing functional that is additively decomposable over disjoint events.

Theorem 4. Suppose that $S^{o}$ has at least three essential disjoint events. Then the following three statements are equivalent.

(i) The weak order $\succeq$ is strictly monotone, supnorm continuous on $\mathcal{F}$, and satisfies the surething principle on $\mathcal{F}_{s}$.

(ii) There exist supnorm continuous functions $U_{A}: \mathcal{F}_{A} \rightarrow[0,1]$ for each event $A$ that are strictly increasing when $A$ is essential and constant when $A$ is null, such that $\succeq$ on $\mathcal{F}$ is represented by

$$
V(f)=\sum_{i=1}^{n} U_{A_{i}}\left(f_{A_{i}}\right)
$$

for each finite partition $\left\{A_{1}, \ldots, A_{n}\right\}$ of events. 
(iii) There exist a (covert) measurable space $\left(S^{c}, \Sigma^{c}\right)$, a finitely additive probability measure $\mathbb{P}$ over an algebra $\mathcal{A}$ on $S^{o} \times S^{c}$ which includes $\mathcal{A}\left(\Sigma^{o} \times \Sigma^{c}\right)$, and an injective benchmark $\beta: S^{c} \rightarrow[a, b]$, such that $\succeq$ on $\mathcal{F}$ is represented by

$$
V(f)=\mathbb{P}(f \geqslant \beta) \text {. }
$$

Moreover, $\mathbb{P}$ is unique; $\mathbb{P}^{o}(A)>0$ if and only if the event $A$ is essential; and $\mathbb{P}^{c}$ on $S^{c}$ (and hence $\mathbb{P}$ itself) are nonatomic.

The equivalence of (i) and (ii) appears as Theorem 11 in Wakker and Zank (1999). Their result establishes the existence of a representing functional for all acts, but derives an explicit functional form only over simple acts. Part (iii), on the other hand, provides an explicit representing functional for all acts. In this respect, the benchmarking procedure seems easier to apprehend.

It should be noted that our proof for the equivalence of (i) and (iii) does not rely on the representation in (ii). We obtain (iii) by applying the Jordan extension technique (described in Appendix A) to the probability measure derived in Theorem 3. As a result, the benchmarking measure in Theorem 4(iii) is the maximally unique extension of the finitely additive probability measure on simple acts obtained in Theorem 3.

\subsection{Pointwise continuity}

In this section, we provide a different extension of the benchmarking representation of Theorem 3 from simple acts to all acts. The extension in Theorem 4 is based on supnorm continuity and gives a finitely additive benchmarking measure for all acts in $\mathcal{F}$. The current extension is based on a stronger pointwise continuity assumption and delivers a countably additive benchmarking measure for all acts in $\mathcal{F}$.

We need a few additional definitions. A sequence $\left\{f_{n}\right\}$ of acts converges pointwise to another act $f$ if $\lim _{n \rightarrow \infty} f_{n}(s)=f(s)$ for any state $s$. The preference relation $\succeq$ on the set $\mathcal{F}$ of all acts is pointwise continuous if, for any sequence $\left\{f_{n}\right\}$ converging pointwise to $f$ and for any act $g$, $f \succ g$ implies $f_{n} \succ g$ for $n$ greater than some $N$ and, similarly, $g \succ f$ implies $g \succ f_{n}$ for $n$ greater than some $N$. Pointwise continuity is equivalent to standard continuity of $\succeq$ when $S^{o}$ is finite, and it implies supnorm continuity.

The third result states that, after exchanging pointwise continuity for supnorm continuity, the benchmarking measure of Theorem 4(iii) becomes countably additive. For symmetry and ease of comparison, we state in (ii) the analog of Theorem 4(ii) asserting the existence of a representing functional which can be written as an integral with respect to a countably additive measure.

Theorem 5. Suppose that $S^{o}$ has at least three essential disjoint events. Then the following three statements are equivalent.

(i) The weak order $\succeq$ is strictly monotone, pointwise continuous on $\mathcal{F}$, and satisfies the surething principle on $\mathcal{F}_{s}$.

(ii) There exists a countably additive probability measure $\mathbb{P}^{o}$ on $S^{o}$ and strictly increasing function $U_{s}: \mathbb{R} \rightarrow[0,1]$ for each state $s$ in $S^{o}$ such that $\succeq$ on $\mathcal{F}$ is represented by

$$
V(f)=\int U_{s}(f(s)) d \mathbb{P}^{o}(s) .
$$


(iii) There exist a (covert) measurable space $\left(S^{c}, \Sigma^{c}\right)$, a countably additive probability measure $\mathbb{P}$ over the $\sigma$-algebra $\sigma \mathcal{A}\left(\Sigma^{o} \times \Sigma^{c}\right)$ on $S^{o} \times S^{c}$, and an injective benchmark $\beta: S^{c} \rightarrow[a, b]$, such that $\succeq$ on $\mathcal{F}$ is represented by

$$
V(f)=\mathbb{P}(f \geqslant \beta) .
$$

Moreover, $\mathbb{P}$ is unique; $\mathbb{P}^{o}(A)>0$ if and only if the event $A$ is essential; and $\mathbb{P}^{c}$ on $S^{c}$ (and hence $\mathbb{P}$ itself ) are nonatomic.

The equivalence of (i) and (ii) appears as Theorem 12 in Wakker and Zank (1999), but our proof is independent of it. We obtain (iii) by applying the Lebesgue extension technique (described in Appendix A) to the probability measure derived in Theorem 3. Using an argument similar to Kindler (1983), we show that under pointwise continuity the benchmarking measure on the semialgebra generated by the subgraphs of simple acts is countably additive. Then the Lebesgue extension provides a countably additive measure over the $\sigma$-algebra generated by the semialgebra. As a result, the benchmarking measure in Theorem 5 is the maximally unique extension of the (now, countably) additive measure on simple acts obtained in Theorem 3.

\subsection{Independent benchmarking}

When the state space is infinite, the link between tradeoff consistency and the factorization of the benchmarking measure into the product of its marginals holds unchanged. The next result modifies the general benchmarking representation for preferences over simple acts of Theorem 3 into the analog of the independent benchmarking representation of Theorem 2 .

Theorem 6. Suppose that $S^{o}$ has at least three essential disjoint events. Then the following three statements are equivalent.

(i) The weak order $\succeq$ is strictly monotone, simply continuous on $\mathcal{F}_{s}$, and satisfies tradeoff consistency on $\mathcal{F}_{S}$.

(ii) There exist a continuous strictly increasing function $U: X \rightarrow[0,1]$ and a finitely additive probability measure $P$ on $\left(S^{o}, \Sigma^{o}\right)$, such that $\succeq$ on $\mathcal{F}_{S}$ is represented by

$$
V\left(\sum_{i=1}^{n} x_{i} \mathbf{1}\left(A_{i}\right)\right)=\sum_{i=1}^{n} U\left(x_{i}\right) P\left(A_{i}\right) .
$$

Moreover, $P(A)>0$ if and only if the event $A$ is essential.

(iii) The statement in Theorem 3(iii) holds. Moreover, $\mathbb{P}=\mathbb{P}^{o} \times \mathbb{P}^{c}$ can be factored in the product of the two marginals; i.e., stochastic independence holds.

The same variation holds for Theorems 4 and 5. This is an immediate corollary of the preceding result, because the representations in Theorems 4 and 5 are based on extensions of a strongly additive set function $m$ initially defined over the (augmented) lattice $\mathcal{G}$ of the subgraphs of the simple acts. When Theorem 6 holds and the measure induced by $m$ over the semialgebra generated by $\mathcal{G}$ decomposes in a product measure, so do its extensions as well. 


\section{Acknowledgments}

We thank Achille Basile, Robert Bordley, Alain Chateauneuf, Christian Gollier, Mark Machina, and especially Fabio Maccheroni and an anonymous referee for their valuable comments. Financial support from MIUR is acknowledged.

\section{Appendix A. Extensions of measures}

A typical problem in measure theory is to extend a measure defined on a collection $\mathcal{C}_{1}$ of sets to a larger collection $\mathcal{C}_{2} \supset \mathcal{C}_{1}$. There is a well-known hierarchy of minimal extension theorems; see for instance Theorem 3.5.1 in Bhaskara Rao and Bhaskara Rao (1983). We collect here the four extension theorems used in this paper. Throughout this section, we use the shorter qualifications "additive" and " $\sigma$-additive" instead of "finitely additive" and "countably additive."

First, suppose that $\mathcal{L}$ is a lattice on $S$ and $m$ is a grounded, positive, and strongly additive set function on $\mathcal{L}$. Let $\mathcal{S}(\mathcal{L})$ be the smallest semialgebra on $S$ containing $\mathcal{L}$; then there exists a unique additive measure $\mu$ on $\mathcal{S}(\mathcal{L})$ which is an extension of $m$ from $\mathcal{L}$ to $\mathcal{S}(\mathcal{L})$. Second, suppose that $\mathcal{S}$ is a semialgebra on $S$ and $m$ is an additive measure on $\mathcal{S}$. Let $\mathcal{A}(\mathcal{S})$ be the smallest algebra on $S$ containing $\mathcal{S}$; then there exists a unique additive measure $\mu$ on $\mathcal{A}(\mathcal{S})$ which is an extension of $m$ from $\mathcal{S}$ to $\mathcal{A}(\mathcal{S})$. The next two extension results deal with non-minimal extensions. Their difference is at the heart of our results, so we describe them less succinctly. See Section 7.27 in Kolmogorov and Fomin (1970) for more details.

Given an algebra $\mathcal{A}$ on $S$ and an additive measure $m$ on $\mathcal{A}$, a subset $A$ of $S$ is Jordan measurable, or $J$-measurable for short, if for any $\varepsilon>0$ there exist two sets $A_{1}, A_{2}$ in $\mathcal{A}$ such that $A_{1} \subseteq A \subseteq A_{2}$ and $m\left(A_{2} \backslash A_{1}\right)<\varepsilon$. The collection $\mathcal{J}(\mathcal{A})$ of all $J$-measurable sets is an algebra that contains the original algebra; that is, $\mathcal{A} \subseteq \mathcal{J}(\mathcal{A})$. For an arbitrary subset $A$ of $S$, define its outer measure by $\mu^{*}(A)=\inf \{m(B): B \supseteq A, B \in \mathcal{A}\}$ and its inner measure by $\mu_{*}(A)=\sup \{m(B): B \subseteq A, B \in \mathcal{A}\}$. Then $\mu_{*}(A) \leqslant \mu^{*}(A)$ for any $A \subseteq S$, with equality holding if and only if $A$ is $J$-measurable. Moreover, $\mu^{*}$ is an additive measure on $\mathcal{J}(\mathcal{A})$. Since $\mu^{*}(A)=m(A)$ for any $A$ in $\mathcal{A}, \mu^{*}$ is called the Jordan extension of $m$ from $\mathcal{A}$ to $\mathcal{J}(\mathcal{A})$.

The Jordan extension $\mu^{*}$ of an additive measure $m$ on an algebra $\mathcal{A}$ is its unique additive extension to the algebra $\mathcal{J}(\mathcal{A})$, in the following sense. A subset $A$ of $S$ is a set of uniqueness for an additive measure $m$ if:

(1) there is an additive extension of $m$ defined on $A$, and

(2) when $\mu_{1}, \mu_{2}$ are two such extensions, then $\mu_{1}(A)=\mu_{2}(A)$.

The collection of sets of uniqueness for an additive measure $m$ on the algebra $\mathcal{A}$ coincides with the algebra $\mathcal{J}(\mathcal{A})$ of the $J$-measurable sets. The additive extension of $m$ to a collection larger than $\mathcal{J}(\mathcal{A})$ is no longer unique. Hence, the Jordan extension is the maximally unique extension of an additive measure on the algebra $\mathcal{A}$.

Given a semialgebra $\mathcal{S}$ on $S$ and a $\sigma$-additive (and finite) measure $m$ on $\mathcal{S}$, define the external measure of an arbitrary subset $A$ of $S$ by

$$
\mu(A)=\inf \left\{\sum_{n=1}^{\infty} m\left(A_{n}\right): A \subseteq \bigcup_{n=1}^{\infty} A_{n},\left\{A_{n}\right\} \subseteq \mathcal{S}\right\} .
$$

A subset $A$ of $S$ is Lebesgue measurable, or $L$-measurable for short, if $\mu(A \cap B)+\mu\left(A^{c} \cap B\right)=$ $m(B)$ for any $B$ in $\mathcal{S}$. The collection $\mathbb{L}(\mathcal{S})$ of all $L$-measurable sets is a $\sigma$-algebra which contains 
the original semialgebra; that is, $\mathcal{S} \subseteq \mathbb{L}(\mathcal{S})$. Since $\mu$ is a $\sigma$-additive measure on $\mathbb{L}(\mathcal{S})$ and $\mu(A)=$ $m(A)$ for any $A$ in $\mathcal{S}, \mu$ is called the Lebesgue extension of $m$ from $\mathcal{S}$ to the $\sigma$-algebra $\mathbb{L}(\mathcal{S})$.

The Lebesgue extension $\mu$ of a $\sigma$-additive measure $m$ on a semialgebra $\mathcal{S}$ is its unique $\sigma$ additive extension to the $\sigma$-algebra $\mathbb{L}(\mathcal{S})$, in the following sense. A subset $A$ of $S$ is a set of $\sigma$-uniqueness for a $\sigma$-additive measure $m$ if:

(1) there is a $\sigma$-additive extension of $m$ defined on $A$, and

(2) when $\mu_{1}, \mu_{2}$ are two such extensions, then $\mu_{1}(A)=\mu_{2}(A)$.

The collection of sets of $\sigma$-uniqueness for a $\sigma$-additive measure $m$ on the semialgebra $\mathcal{S}$ coincides with the $\sigma$-algebra $\mathbb{L}(\mathcal{S})$ of the $L$-measurable sets. Hence, the Lebesgue extension is the maximally unique extension of a $\sigma$-additive measure on the semialgebra $\mathcal{S}$. Finally, we note that any $J$-measurable set $A$ is also $L$-measurable and $\mu^{*}(A)=\mu(A)$. Thus, $\mathcal{J}[\mathcal{A}(\mathcal{S})] \subseteq \mathbb{L}(\mathcal{S})$ and the Jordan extension of a $\sigma$-additive measure $m$ on a semialgebra $\mathcal{S}$ is also $\sigma$-additive.

\section{Appendix B. Proofs}

Proof of Theorem 1. The equivalence of (i) and (ii) is Theorem 3 in Debreu (1960), where it is also shown that another functional $W(f)=\sum_{i} W_{i}\left(f\left(s_{i}\right)\right)$ represents $\succeq$ if and only if there exist real numbers $\alpha_{1}, \ldots, \alpha_{n}$ and a strictly positive $\gamma$ such that $W_{i}=\alpha_{i}+\gamma U_{i}$ for all $i$, implying that $W=\sum_{i} \alpha_{i}+\gamma V$.

We prove that (ii) implies (iii). Replacing (if necessary) $U_{i}(x)$ with $U_{i}(x)-U_{i}(a)$, assume $U_{i}(a)=0$ for all $i$. Hence, $V(a)=0$; without loss of generality, let $V(b)=1$. For each essential state $s_{i}, U_{i}(x)$ is continuous, strictly increasing and bounded above by $V(b)=1$. Therefore, it induces a unique (countably additive and) strictly positive measure $\mu_{i}$ on the Borel $\sigma$-algebra $\mathcal{B}([a, b])$. Now $\mu_{i}$ generates a unique (countably additive and) strictly positive measure $v_{i}$ on $\left(S^{o} \times[a, b], \Sigma^{o} \times \mathcal{B}([a, b])\right.$ defined by $v_{i}(A)=\mu_{i}\left(A \cap\left(\left\{s_{i}\right\} \times[a, b]\right)\right)$. The abuse in notation is justified by the identification of $\mathcal{B}([a, b])$ with $\left\{s_{i}\right\} \times \mathcal{B}([a, b])$. Clearly, the measure $v_{i}$ is nonatomic: if $\left(s_{j}, x\right) \notin\left\{s_{i}\right\} \times[a, b]$, then $v_{i}\left(\left\{\left(s_{j}, x\right)\right\}\right)=0$; else, if $s_{j}=s_{i}$, then

$$
v_{i}\left(\left\{\left(s_{j}, x\right)\right\}\right)=v_{i}\left(\left\{s_{i}\right\} \times\{x\}\right)=\mu_{i}(\{x\})=U_{i}(x)-\lim _{y \uparrow x} U_{i}(y)=0
$$

by continuity of $U_{i}$. On the other hand, for each null state $s_{i}, U_{i}(x)$ is constant and by a similar construction induces a null measure. Since

$$
\sum_{i=1}^{n} v_{i}\left(S^{o} \times[a, b]\right)=\sum_{i=1}^{n} \mu_{i}([a, b])=\sum_{i=1}^{n} U_{i}(b)=1,
$$

it follows that $\mathbb{P}=\sum_{i} \mu_{i}$ is a nonatomic (and finitely additive) probability measure on ( $S^{o} \times$ $\left.[a, b], \Sigma^{o} \times \mathcal{B}([a, b])\right)$. Finally, choose $S^{c}=[a, b]$ and define the random variable $\beta(s)=s$ to obtain

$$
\begin{aligned}
\mathbb{P}(f \geqslant \beta) & =\mathbb{P}\left(\left\{\left(s^{o}, s^{c}\right) \in S^{o} \times S^{c}: f\left(s^{o}\right) \geqslant \beta\left(s^{c}\right)\right\}\right) \\
& =\mathbb{P}\left(\left\{\left(s^{o}, s^{c}\right) \in S^{o} \times S^{c}: s^{c} \leqslant f\left(s^{o}\right)\right\}\right) \\
& =\sum_{i=1}^{n} v_{i}\left(\left\{\left(s^{o}, s^{c}\right) \in S^{o} \times S^{c}: s^{c} \leqslant f\left(s^{o}\right)\right\}\right)
\end{aligned}
$$




$$
\begin{aligned}
& =\sum_{i=1}^{n} \mu_{i}\left(\left\{\left(s^{o}, s^{c}\right) \in S^{o} \times S^{c}: s^{c} \leqslant f\left(s^{o}\right)\right\} \cap\left(\left\{s_{i}\right\} \times[a, b]\right)\right) \\
& =\sum_{i=1}^{n} \mu_{i}\left(\left\{\left(s^{o}, s^{c}\right) \in\left\{s_{i}\right\} \times S^{c}: s^{c} \leqslant f\left(s_{i}\right)\right\}\right) \\
& =\sum_{i=1}^{n} \mu_{i}\left(\left[a, f\left(s_{i}\right)\right]\right)=\sum_{i=1}^{n} U_{i}\left(f\left(s_{i}\right)\right) .
\end{aligned}
$$

We prove that (iii) implies (ii). Since

$$
\begin{aligned}
\mathbb{P}(f \geqslant \beta) & =\mathbb{P}\left(\left\{\left(s^{o}, s^{c}\right) \in S^{o} \times S^{c}: f\left(s^{o}\right) \geqslant \beta\left(s^{c}\right)\right\}\right) \\
& =\sum_{i=1}^{n} \mathbb{P}\left(\left\{\left(s^{o}, s^{c}\right) \in\left\{s_{i}\right\} \times S^{c}: \beta\left(s^{c}\right) \leqslant f\left(s_{i}\right)\right\}\right),
\end{aligned}
$$

define for each $i=1, \ldots, n$

$$
U_{i}(t)=\mathbb{P}\left(\left\{\left(s^{o}, s^{c}\right) \in\left\{s_{i}\right\} \times S^{c}: \beta\left(s^{c}\right) \leqslant t\right\}\right)
$$

and $V(f)=\sum_{i} U_{i}\left(f\left(s_{i}\right)\right)$. Then $V(f)$ represents $\succeq$. Moreover, $U_{i}(t)$ is strictly increasing (constant, respectively) if and only if the state $S_{i}$ is essential (null). It remains to show that $U_{i}$ is continuous. This follows because the measure on $\left(S^{c}, \Sigma^{c}\right)$ defined by $\mu_{i}(A)=\mathbb{P}\left(\left\{s_{i}\right\} \times A\right)$ is nonatomic and $U_{i}(t)=\mu_{i}\left(s^{c} \in S^{c}: \beta(s) \leqslant t\right)$ is the distribution function of an injective random variable with respect to a nonatomic measure.

Proof of Theorem 2. The equivalence of (i) and (ii) follows from Lemma 24 and Corollary 10 in Köbberling and Wakker (2003), with the addition that the strict monotonicity of $\succeq$ implies that $U$ is strictly increasing. The utility function $U$ is unique up to a strictly increasing affine transformation and the probability measure $P$ is uniquely determined.

We prove that (ii) implies (iii). Define $S^{c}=[a, b]$ and let $\Sigma^{c}$ be the Borel $\sigma$-algebra $\mathcal{B}([a, b])$. Assume without loss of generality $U(a)=0$ and $U(b)=1$. Since $U$ is continuous and strictly increasing, it is the distribution function of a random variable $\beta(s)=s$ which induces a unique strictly positive and countably additive measure $\mathbb{P}^{c}$ on $\Sigma^{c}$.

Define the finitely additive probability measure $\mathbb{P}^{o}=P$ on $\left(S^{o}, \Sigma^{o}\right)$. Finally, let $\mathbb{P}=\mathbb{P}^{o} \times \mathbb{P}^{c}$ over the algebra $\mathcal{A}\left(\Sigma^{o} \times \Sigma^{c}\right)$ on $S^{o} \times S^{c}$. For any simple act $f=\sum_{i=1}^{n} x_{i} \mathbf{1}\left(A_{i}\right)$, we have

$$
\begin{aligned}
\mathbb{P}(f \geqslant \beta) & =\mathbb{P}\left(\left\{\left(s^{o}, s^{c}\right) \in S^{o} \times S^{c}: \beta\left(s^{c}\right) \leqslant f\left(s^{o}\right)\right\}\right) \\
& =\sum_{i=1}^{n} \mathbb{P}^{c}\left(\left\{s^{c} \in S^{c}: \beta\left(s^{c}\right) \leqslant x_{i}\right\}\right) \cdot \mathbb{P}^{o}\left(\left\{s^{o} \in S^{o}: f\left(s^{o}\right)=x_{i}\right\}\right) \\
& =\sum_{i=1}^{n} U\left(x_{i}\right) P\left(A_{i}\right) \\
& =V\left(\sum_{i=1}^{n} x_{i} \mathbf{1}\left(A_{i}\right)\right) .
\end{aligned}
$$

The proof that (iii) implies (ii) is analogous to the proof of Theorem 1 and follows by reading the equalities backward. 
Proof of Theorem 3. The implication from (i) to (ii) is Proposition 3 in Wakker and Zank (1999), where it is also shown that another functional $W\left(\sum_{i=1}^{n} x_{i} \mathbf{1}\left(A_{i}\right)\right)=\sum_{i=1}^{n} U_{A_{i}}\left(x_{i}\right)$ represents $\succeq$ on $\mathcal{F}_{S}$ if and only if there exist real numbers $\alpha_{A}$ for each event $A$ in $\Sigma^{o}$ and a strictly positive $\gamma$ such that $W_{A}=\alpha_{A}+\gamma U_{A}$ for all $A$ in $\Sigma^{o}$ with $\alpha_{A \cup B}=\alpha_{A}+\alpha_{B}$ for all disjoint events, implying that $W=\alpha_{S^{o}}+\gamma V$. The implication from (ii) to (i) is obvious.

The proof that (iii) implies (ii) is analogous to that one given for Theorem 1, so we only prove that (ii) implies (iii). Replacing (if necessary) $U_{A}(x)$ with $U_{A}(x)-U_{A}(a)$ for any event $A$, assume $U_{A}(a)=0$ for all $i$. Hence, $V(a)=0$; without loss of generality, let $V(b)=1$. Recall that an act $f$ is a function from $S^{o}$ in $X=[a, b]$. Identify each simple act $f$ in $\mathcal{F}_{s}$ with its (truncated) subgraph $\hat{f}=\left\{(s, x) \in S^{o} \times[a, b]: a \leqslant x \leqslant f(s)\right\}$. There is an obvious 1-1 onto mapping between the set $\mathcal{F}_{s}$ of simple acts and the set $\mathcal{G}_{0}$ of their subgraphs.

Lemma B.1. The set $\mathcal{G}_{0}$ of subgraphs of simple functions is a lattice.

Proof. Clearly, $S=\hat{b} \in \mathcal{G}_{0}$. Now, let $\hat{f}$ and $\hat{g}$ be the subgraphs of two simple acts $f=$ $\sum_{i=1}^{n} x_{i} \mathbf{1}\left(A_{i}\right)$ and $g=\sum_{i=1}^{m} y_{i} \mathbf{1}\left(B_{i}\right)$. We need to show that $\hat{f} \cup \hat{g}$ and $\hat{f} \cap \hat{g}$ are also subgraphs of simple functions. Let $\Pi=\left\{C_{1}, \ldots, C_{k}\right\}$ be the coarsest common refinement of the two partitions $\left\{A_{1}, \ldots, A_{n}\right\}$ and $\left\{B_{1}, \ldots, B_{m}\right\}$. Clearly, we can write $f=\sum_{i=1}^{k} x_{i} \mathbf{1}\left(C_{i}\right)$ and $g=\sum_{i=1}^{k} y_{i} \mathbf{1}\left(C_{i}\right)$ as simple functions on $\Pi$. But then $\hat{f} \cup \hat{g}$ is the subgraph of the simple function $(f \vee g)=$ $\sum_{i=1}^{k}\left(x_{i} \vee y_{i}\right) \mathbf{1}\left(C_{i}\right)$. Similarly, $\hat{f} \cap \hat{g}$ is the subgraph of $(f \wedge g)=\sum_{i=1}^{k}\left(x_{i} \wedge y_{i}\right) \mathbf{1}\left(C_{i}\right)$.

The functional $V$ on $\mathcal{F}_{s}$ defines a set function $m$ on the lattice $\mathcal{G}_{0}$ by $m(\hat{f})=V(f)$. Note that $m(\hat{a})=V(a)=0$ and $m(\hat{b})=V(b)=1$.

Lemma B.2. The set function $m$ on the lattice $\mathcal{G}_{0}$ is strongly additive.

Proof. Let $\hat{f}$ and $\hat{g}$ be the subgraphs of two simple acts $f=\sum_{i=1}^{n} x_{i} \mathbf{1}\left(A_{i}\right)$ and $g=$ $\sum_{i=1}^{m} y_{i} \mathbf{1}\left(B_{i}\right)$. We need to show that $m(\hat{f})+m(\hat{g})=m(\hat{f} \cup \hat{g})+m(\hat{f} \cap \hat{g})$. Replacing $\left\{A_{1}, \ldots, A_{n}\right\}$ and $\left\{B_{1}, \ldots, B_{m}\right\}$ with their coarsest common refinement $\left\{C_{1}, \ldots, C_{k}\right\}$, we can assume that $f=\sum_{i=1}^{k} x_{i} \mathbf{1}\left(C_{i}\right)$ and $g=\sum_{i=1}^{k} y_{i} \mathbf{1}\left(C_{i}\right)$. Then

$$
\begin{aligned}
m(\hat{f})+m(\hat{g}) & =V(f)+V(g) \\
& =\sum_{i=1}^{n} U_{C_{i}}\left(x_{i}\right)+\sum_{i=1}^{n} U_{C_{i}}\left(y_{i}\right) \\
& =\sum_{i=1}^{n} U_{C_{i}}\left(x_{i} \vee y_{i}\right)+\sum_{i=1}^{n} U_{C_{i}}\left(x_{i} \wedge y_{i}\right) \\
& =V(f \vee g)+V(f \wedge g) \\
& =m(\hat{f} \cup \hat{g})+m(\hat{f} \cap \hat{g}) .
\end{aligned}
$$

The set function $m$ on the lattice $\mathcal{G}_{0}$ trivially extends to the augmented lattice $\mathcal{G}=\mathcal{G}_{0} \cup\{\emptyset\}$ by setting $m(\emptyset)=0$. Clearly, the set function $m$ on the lattice $\mathcal{G}$ is grounded, positive, and strongly additive and thus, by Theorem 3.5.1(iii) in Bhaskara Rao and Bhaskara Rao (1983), it has a unique extension to a finitely additive and positive measure $m^{\prime}$ on the semialgebra $\mathcal{S}(\mathcal{G})=\{A \backslash B: A, B \in \mathcal{G}, B \subseteq A\}$ generated by $\mathcal{G}$. This unique extension obtains by defining $m^{\prime}(A \backslash B)=m(A)-m(B)$ for any $A, B$ in $\mathcal{G}$ with $B \subseteq A$. 
By Theorem 3.5.1(ii) in Bhaskara Rao and Bhaskara Rao (1983), the measure $m^{\prime}$ has a unique extension to a finitely additive and positive measure $\mu$ on the algebra $\mathcal{A}[\mathcal{S}(\mathcal{G})]=$ $\left\{\bigcup_{i=1}^{n} C_{i}: C_{i} \in \mathcal{S}, C_{i} \cap C_{j}=\emptyset\right.$ for $\left.i \neq j\right\}$ generated by $\mathcal{S}(\mathcal{G})$. This unique extension obtains by defining $\mu\left(\bigcup_{i=1}^{n} C_{i}\right)=\sum_{i=1}^{n} m^{\prime}\left(C_{i}\right)$ for any finite union of disjoint sets $C_{1}, \ldots, C_{n}$ from $\mathcal{S}(\mathcal{G})$.

Since $\mu(\hat{b})=m(\hat{b})=1, \mu$ is a (finitely additive) probability measure on the algebra $\mathcal{A}[\mathcal{S}(\mathcal{G})]$. The benchmarking measure can be obtained from $\mu$.

\section{Lemma B.3. The finitely additive probability measure $\mu$ is a benchmarking measure.}

Proof. Choose $S^{c}=[a, b]$ and let $\Sigma^{c}$ be the semialgebra formed by all the clopen intervals $(y, x]$ and all the closed intervals $[a, x]$ in $[a, b]$. Then the product semialgebra $\Sigma^{o} \times \Sigma^{c}$ coincides with $\mathcal{S}(\mathcal{G})$ and thus $\mu$ defines a finitely additive probability measure $\mathbb{P}$ on the algebra $\mathcal{A}\left(\Sigma^{o} \times \Sigma^{c}\right)$ on $S^{o} \times S^{c}$. Choosing $\beta(s)=s$ as benchmark, we have $\mathbb{P}(f \geqslant \beta)=\mathbb{P}\left(\left\{\left(s^{o}, s^{c}\right) \in\right.\right.$ $\left.\left.S^{o} \times S^{c}: f\left(s^{o}\right) \geqslant \beta\left(s^{c}\right)\right\}\right)=m(\hat{f})$ and thus $\mathbb{P}$ is a benchmarking measure.

Finally, consider the two marginal probability measures induced by $\mathbb{P}$. First, since $U_{A}$ is strictly increasing if the event $A$ in $\Sigma^{o}$ is essential and constant otherwise, it follows that $\mathbb{P}^{o}(A)=m\left(b \mathbf{1}(A)+a \mathbf{1}\left(S^{o} \backslash A\right)\right)=U_{A}(b)$ is strictly greater than $U_{A}(a)=0$ when $A$ is essential, and it is equal to $U_{A}(a)=0$ otherwise. Second, note that $\mathbb{P}^{c}((y, x])=U_{S^{o}}(x)-U_{S^{o}}(y)$ for $y<x$; since, $U_{S^{o}}$ is continuous and strictly increasing, for $y<x$ we have $\mathbb{P}^{c}((y, x])>0$ and $\mathbb{P}^{c}((y, x]) \downarrow 0$ as $y \uparrow x$; therefore, $\mathbb{P}^{c}$ is nonatomic. This concludes the proof of Theorem 3 .

Proof of Theorem 4. The equivalence of (i) and (ii) is Theorem 11 in Wakker and Zank (1999). The proof that (iii) implies (i) is straightforward. We only prove that (i) implies (iii).

The supnorm continuity of the preference relation implies its simple continuity. Therefore, the assumptions in Theorem 3(i) are satisfied and we can replicate its proof (up to just before Lemma B.3) to derive the existence of a finitely additive measure $\mu$ on the algebra $\mathcal{A}=\mathcal{A}[\mathcal{S}(\mathcal{G})]$ generated by $\mathcal{S}(\mathcal{G})$.

The algebra $\mathcal{A}$ is the smallest algebra generated by $\mathcal{G}$. We uniquely extend $\mu$ to a measure $\mu^{J}$ on the (possibly) larger algebra $\mathcal{A}^{J}$ of the $J$-measurable sets using the Jordan extension described in Appendix A. Under the supnorm continuity of $\succeq$, the subgraph $\hat{f}$ of an arbitrary act $f$ in $\mathcal{F}$ is $J$-measurable.

\section{Lemma B.4. The subgraph $\hat{f}$ of any act $f$ is $J$-measurable.}

Proof. The set $\mathcal{F}_{s}$ of simple functions is supnorm dense in $\mathcal{F}$. Therefore, for any act $f$ in $\mathcal{F}$, we can find two sequences of simple acts $\left\{g_{i}\right\}$ and $\left\{h_{i}\right\}$ such that $g_{i}$ and $h_{i}$ converge in supnorm to $f$ with $g_{i}(s) \leqslant g_{i+1}(s) \leqslant f(s) \leqslant h_{i+1}(s) \leqslant h(s)$ for any state $s$ in $S^{o}$. Recall from Appendix A the definitions of inner and outer measure. Then $\mu\left(\hat{g}_{i}\right) \leqslant \mu_{*}(\hat{f}) \leqslant \mu^{*}(\hat{f}) \leqslant \mu\left(\hat{h}_{i}\right)$ for all $i$. By supnorm continuity, $\mu\left(\hat{h}_{i}\right)-\mu\left(\hat{g}_{i}\right) \downarrow 0$. Therefore, $\mu_{*}(\hat{f})=\mu^{*}(\hat{f})$ and hence the subgraph $\hat{f}$ of any act $f$ is $J$-measurable.

This $J$-measurability implies that the Jordan extension $\mu^{J}$ of $\mu$ to $\mathcal{A}^{J} \supseteq \mathcal{A}$ is well defined for the subgraph $\hat{f}$ of any act $f$ in $\mathcal{F}$. The rest of the proof goes on as for Theorem 3. In particular, apply Lemma B.3 to $\mu^{J}$ on $\mathcal{A}^{J}$ to obtain the benchmarking measure. This concludes the proof of Theorem 4. 
Proof of Theorem 5. The equivalence of (i) and (ii) is Theorem 12 in Wakker and Zank (1999). The proof that (iii) implies (i) is straightforward. We only prove that (i) implies (iii).

The pointwise continuity of the preference relation implies its simple continuity. Therefore, the assumptions in Theorem 3(i) are satisfied and we can replicate its proof (up to right after Lemma B.2) to derive the existence of a finitely additive measure $\mu$ on the semialgebra $\mathcal{S}(\mathcal{G})=\{A \backslash B: A, B \in \mathcal{G}, B \subseteq A\}$ generated by the lattice of the subgraphs of the simple acts in $\mathcal{F}_{S}$, augmented with the empty set. For any pair of simple acts $f \leqslant g$, define the set $(f, g]=\left\{\left(s^{o}, s^{c}\right) \in S^{o} \times S^{c}: f\left(s^{o}\right)<s^{c} \leqslant g\left(s^{o}\right)\right\}$; the set $[f, g]$ is defined analogously. Clearly, $\hat{g} \backslash \hat{f}=(f, g]$ and therefore the semialgebra $\mathcal{S}(\mathcal{G})$ is formed by the collection of all sets $(f, g]$ and $[a, f]$, for $a \leqslant f \leqslant g$ in $\mathcal{F}_{s}$. Under the pointwise continuity of $\succeq$, the measure $\mu$ on the semialgebra $\mathcal{S}(\mathcal{G})$ is countably additive.

Lemma B.5. The measure $\mu$ on the semialgebra $\mathcal{S}(\mathcal{G})$ is countably additive.

Proof. For $f \leqslant g$ in $\mathcal{F}_{s}$, suppose without loss of generality that $(f, g]=\bigcup_{n}\left(f_{n}, g_{n}\right]$ can be written as a countable union of disjoint sets $\left(f_{n}, g_{n}\right]$, where $f_{n}, g_{n}$ are simple acts for all $n$. We need to show that $\mu((f, g])=\sum_{n} \mu\left(\left(f_{n}, g_{n}\right]\right)$. For any $s^{o}$ in $S^{o},\left(f\left(s^{o}\right), g\left(s^{o}\right)\right]=\bigcup_{n}\left(f_{n}\left(s^{o}\right), g_{n}\left(s^{o}\right)\right]$. Since the length of real-valued intervals is countably additive, it follows that $g\left(s^{o}\right)-f\left(s^{o}\right)=$ $\sum_{n}\left[g_{n}\left(s^{o}\right)-f_{n}\left(s^{o}\right)\right]$ for any $s^{o}$. Define $h_{n}=a+\left[g-f-\sum_{i=1}^{n}\left(g_{i}-f_{i}\right)\right]$. Then $h_{n} \in \mathcal{F}_{s}$ for all $n$ and $h_{n}$ pointwise converges to $a$. By pointwise continuity, $\mu\left(\hat{h}_{n}\right) \downarrow \mu(\hat{a})=0$ and thus $\mu((f, g])=\sum_{n} \mu\left(\left(f_{n}, g_{n}\right]\right)$.

Since $\mu(\hat{b})=m(\hat{b})=1, \mu$ is finite. Therefore, this countable additivity implies that we can uniquely extend $\mu$ from the semialgebra $\mathcal{S}(\mathcal{G})$ to a measure $\mu^{L}$ on the $\sigma$-algebra $\sigma \mathcal{A}\left(\Sigma^{0} \times\right.$ $\Sigma^{c}$ ) using the Lebesgue extension described in Appendix A. Any $J$-measurable set is also $L$ measurable and thus the Lebesgue extension $\mu^{L}$ is a countably additive measure on $\sigma \mathcal{A}\left(\Sigma^{0} \times\right.$ $\left.\Sigma^{c}\right) \supseteq \mathcal{A}^{J} \supseteq \mathcal{S}(\mathcal{G})$ such that $\mu^{L}(A)=\mu(A)$ for any event $A$ in the semialgebra $\mathcal{S}(\mathcal{G})$. The proof concludes by applying Lemma B.3 to $\mu^{L}$ on $\sigma \mathcal{A}\left(\Sigma^{0} \times \Sigma^{c}\right)$.

Proof of Theorem 6. The proof of the equivalence of (i) and (ii) is sketched in Section 5.1 of Köbberling and Wakker (2003) and requires a trivial modification of the argument given in Section 5.3 of Wakker (1989). The proof that (ii) implies (iii) is formally identical to the corresponding argument in Theorem 3, except for that the right-hand term in the definition $m(\hat{f})=V(f)$ is a different functional and thus a single normalization $U(a)=0$ and $U(b)=1$ suffices. The proof that (iii) implies (ii) is analogous to that one given for Theorem 2.

\section{References}

Bhaskara Rao, K.P.S., Bhaskara Rao, M., 1983. Theory of Charges. Academic Press, London.

Bordley, R.F., 2002. Foundations of target-based decision theory. In: Brachinger, H.W., Monney, P.A. (Eds.), In: Decision Analysis. From: Encyclopedia of Life Support Systems (EOLSS). Eolss Publishers, Oxford. Available from: http: //www.eolss.net.

Bordley, R.F., LiCalzi, M., 2000. Decision analysis using targets instead of utility functions. Dec. Econ. Finance 23, 53-74.

Castagnoli, E., LiCalzi, M., 1996. Expected utility without utility. Theory Dec. 41, 281-301.

Chateauneuf, A., 1999. Comonotonicity axioms and rank-dependent expected utility theory for arbitrary consequences. J. Math. Econ. 32, 21-45.

Chew, S.H., Wakker, P., 1996. The comonotonic sure-thing principle. J. Risk Uncertainty 12, 5-27. 
Debreu, G., 1960. Topological methods in cardinal utility theory. In: Arrow, K.J., Karlin, S., Suppes, P. (Eds.), Mathematical Methods in the Social Sciences. Stanford Univ. Press, Stanford, pp. 16-26.

Gorman, W.M., 1968. The structure of utility functions. Rev. Econ. Stud. 35, 367-390.

Karni, E., Safra, Z., 1998. The hexagon condition and additive representation for two dimensions: An algebraic approach. J. Math. Psych. 42, 393-399.

Kindler, J., 1983. A simple proof of the Daniel-Stone representation theorem. Amer. Math. Mon. 90, $396-397$.

Köbberling, V., 2003. Comments on: Edi Karni and Zvi Safra (1998). The hexagon condition and additive representation for two dimensions: An algebraic approach. J. Math. Psych. 47, 370.

Köbberling, V., Wakker, P., 2003. Preference foundations for nonexpected utility: A generalized and simplified technique. Math. Operations Res. 28, 395-423.

Kolmogorov, A.N., Fomin, S.V., 1970. Introductory Real Analysis. Prentice Hall International, Englewood Cliffs, NJ.

LiCalzi, M., 1998. Variations on the measure representation approach. J. Math. Econ. 29, 255-269.

Savage, L.J., 1954. The Foundations of Statistics. Wiley, New York.

Segal, U., 1989. Anticipated utility: A measure representation approach. Ann. Operations Res. 19, 359-373.

Segal, U., 1993. The measure representation: A correction. J. Risk Uncertainty 6, 99-107.

Wakker, P., 1984. Cardinal coordinate independence for expected utility. J. Math. Psych. 28, 110-117.

Wakker, P., 1989. Additive Representations of Preferences. Kluwer, Dordrecht.

Wakker, P., 1993. Counterexamples to Segal's measure representation theorem. J. Risk Uncertainty 6, 91-98.

Wakker, P., Zank, H., 1999. State dependent expected utility for Savage's state space. Math. Operations Res. 24 , 8-34. 